James Madison University JMU Scholarly Commons

Department of Mathematics and Statistics - Faculty

Scholarship

Department of Mathematics and Statistics

2015

\title{
On Some Recent Experimental Results Concerning Turbulent Coanda Wall Jets
}

Caroline P. Lubert

James Madison University, lubertcp@jmu.edu

Follow this and additional works at: http://commons.lib.jmu.edu/mathstat

Part of the Aerospace Engineering Commons, and the Applied Mathematics Commons

\section{Recommended Citation}

Lubert, C. P. (2014, October). Some recent experimental results concerning turbulent Coanda wall jets. In Proceedings of Meetings on Acoustics (Vol. 22, No. 1, p. 040004). ASA. https://doi.org/10.1121/2.0000040

This Article is brought to you for free and open access by the Department of Mathematics and Statistics at JMU Scholarly Commons. It has been accepted for inclusion in Department of Mathematics and Statistics - Faculty Scholarship by an authorized administrator of JMU Scholarly Commons.

For more information, please contact dc_admin@jmu.edu. 


\title{
SOME RECENT EXPERIMENTAL RESULTS CONCERNING TURBULENT COANDA WALL JETS \\ Caroline P. Lubert, Department of Mathematics \& Statistics, James Madison University, Harrisonburg, VA.
}

\begin{abstract}
The Coanda effect is the tendency of a stream of fluid to stay attached to a convex surface, rather than follow a straight line in its original direction. As a result, in such jets mixing takes place between the jet and the ambient air as soon as the jet issues from its exit nozzle, causing air to be entrained. This air-jet mixture adheres to the nearby surface. Whilst devices employing the Coanda effect usually offer substantial flow deflection, and enhanced turbulence levels and entrainment compared with conventional jet flows, these prospective advantages are generally accompanied by significant disadvantages including a considerable increase in associated noise levels and jet breakaway. Generally, the reasons for these issues are not well understood and thus the full potential offered by the Coanda effect is yet to be realized. The development of a model for predicting the noise emitted by three-dimensional flows over Coanda surfaces would suggest ways in which this noise could be reduced or attenuated. In this paper, the results of recent modelling and experiments on a 3-D turbulent Coanda wall jet are presented. They include the relationship of SPL, shock cell distribution and breakaway to various flow parameters, and predictions of the jet boundary. The potential application of these results to important problems of practical interest such as launch noise is also discussed, and recent data pertaining to rocket launch noise sources is presented.
\end{abstract}

\section{INTRODUCTION}

\subsection{Coanda Flows}

The Coanda effect was discovered early in the twentieth century by Romanian mathematician and scientist Henri Coanda, who described it as the phenomenon whereby “...when a jet is passed over a curved surface it bends to follow the surface, entraining large amounts of air as it does so..." [1]. Consider a fluid element exiting a nozzle adjacent to a curved surface. The radial equilibrium of the element leads to the development of a pressure field which forces the fluid against the surface, and this effect is reinforced by the slightly enhanced viscous drag which is experienced by the jet on its wall side as it exits the nozzle, and which also tends to deflect it towards the wall. Subsequently, this pressure field will continue to force the jet towards the surface. An additional viscous effect, namely the entrainment of the ambient fluid between the jet and the surface, may also help to move the jet towards the wall. The effect breaks down under certain operating conditions, at which point jet breakaway occurs. A hysteresis effect is subsequently observed.

The Coanda effect is noticed in the natural world (with both positive and negative consequences) and is frequently invoked in aeronautics, maritime technology and industrial engineering [2]. The substantial flow deflection offered by the Coanda principle is generally accompanied by enhanced levels of turbulence and increased entrainment. A related, although generally undesirable, side-effect is the significant increase in associated noise levels. It is suggested [3] that this has prevented the Coanda effect from being more widely applied. The present work is part of an effort to better understand the mechanisms behind the aerodynamic noise generation in such jets, with the goal of facilitating improvements in the prediction and attenuation of such noise. 


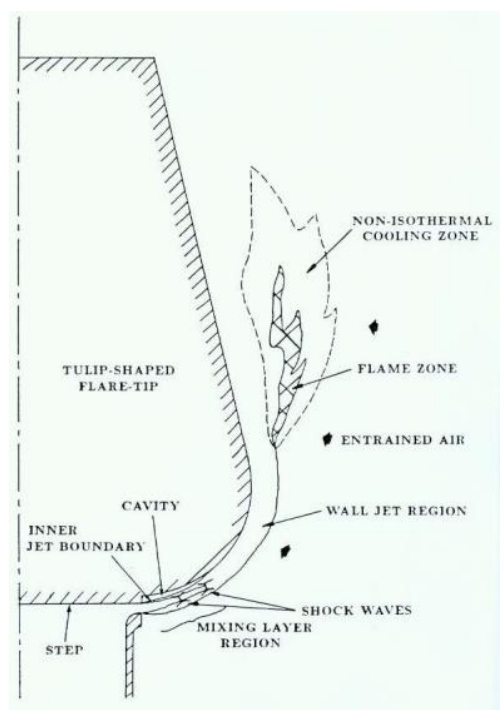

Figure 1. The flow field and combustion zone of a Coanda Flare

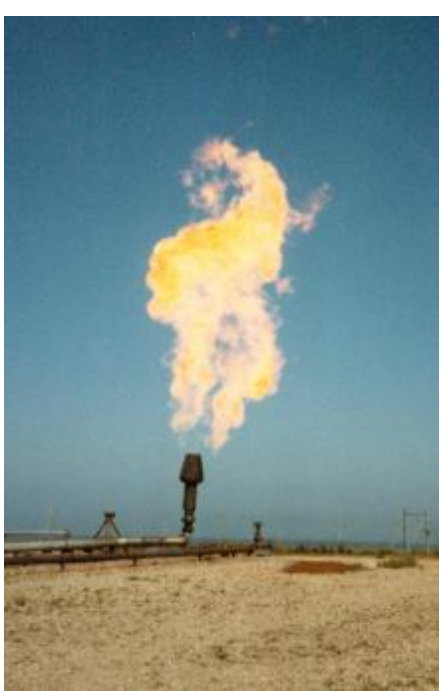

Figure 2. An operating Coanda flare

The jet under consideration here is assumed to issue at high velocity from an annular exit slot. Immediately upon exit it is adjacent to a solid three-dimensional Coanda surface. The geometry of interest is shown in Figure 1. Although this representation is that of a Coanda flare $[4,5]$ of the type used in the petroleum industry (an example of which is shown in operation in Figure 2), the experimental results and models developed herein can easily be applied to other examples of three-dimensional turbulent jet flows over Coanda surfaces.

\subsection{Coanda Wall Jet Noise Generation}

Jet flows such as that shown in Figure 1 emit both low- and high-frequency noise. It is the latter that is of greatest interest, since it is both the most annoying to the human ear, and the easiest to attenuate. There are two primary high-frequency noise sources; turbulent mixing noise (TMN) and shock-associated noise (SAN). This paper describes both types of noise in detail. A comparison of the phenomena for Coanda jets and plane or axisymmetric jets is also given.

\subsubsection{Turbulent Mixing Noise (TMN)}

The flow field and combustion zone of the Indair flare is shown in Figure 1. Immediately the flow leaves the nozzle exit, it entrains the ambient air, causing turbulent mixing to take place between the two. Owing to the Coanda effect, this air-gas mixture then adheres to the flare-tip surface, causing further entrainment, and turbulent shear layers are thus formed in the mixinglayer and wall-jet regions of the flare flow field. These turbulent shear layers play an important part in the various noise-generation mechanisms responsible for the high-frequency component of flare noise. In particular, the convection downstream of large eddies, or large-scale coherent structures present in the flare jet, by the mean jet flow, causes fluctuations in the turbulent shear stress in these shear layers. The kinetic energy of the fluctuating stress is converted to acoustic energy, and the aerodynamic noise thus generated is known as Turbulent Mixing Noise. Since a fluctuating stress field is physically equivalent to a distribution of quadrupole-type acoustic sources, in order to be able to investigate this type of wall jet noise, it is therefore necessary to clarify the effects of the solid surface on the sound generated by such sources. Clearly a curved turbulent wall jet flow field is very complicated. Radial expansions, shock waves, expansion waves and surface curvature effects are all present. Accordingly, the much simpler problem of a plane two-dimensional wall-jet has first been investigated. The ideas and methods developed to deal with this problem can then be extended to the case of a curved radial wall-jet. This will allow more accurate modelling of the turbulent mixing noise associated with Coanda flows than has previously been achieved. 
The precise problem under consideration is that of calculating the sound radiated to the far-field by unit volume of jet-type shear-layer turbulence in close proximity to an infinite rigid plane. It is thus necessary to find some method of estimating the sound radiated by a turbulent fluid flow. There are essentially two approaches to the problem. The first is based on theory developed by Lighthill [6], in which he drew an acoustic analogy between the equation of sound in a stationary medium, and a wave equation derived from the exact equations of fluid motion. Since it is actually the sound radiated by unit volume of jet-type shear-layer turbulence that is being modelled, a more appropriate analogy, however, would seem to be the one drawn by Lilley [7], between this wave equation, and the equation of sound in a moving medium.

Comparing results obtained by means of both theories with experimental data for a plane two-dimensional jet, Ribner [8] has shown that the solution obtained from Lilley's equation is slightly more accurate. The loss of accuracy using Lighthill's approach occurs mainly in a region known as the 'Refraction Valley', where the refraction of sound by the jet is a maximum. This is because, although Lighthill's theory is exact, and in principle an exact solution to it exists, in practice simplifying assumptions such as neglecting refraction effects need to be made, in order to obtain a solution. Lilley's equation, on the other hand, is a convected wave equation, which has the capability of dealing with the effects of refraction on the jet noise directivity. However, Lilley's theory is not exact, and must be solved numerically. Therefore, the slight loss of accuracy which occurs using Lighthill's theory is not considered important, compared to the far greater simplicity of solution with this approach. Thus a theory has been developed for estimating the Turbulent Mixing Noise emitted by unit volume of jet-type shear-layer turbulence in close proximity to an infinite rigid plane [9-15] based on Lighthill's [6] theory. This theory was applied to unit volume of free field turbulence, and an extension of this theory, developed by Curle [16] and Powell [17] was used to take into account the presence of the rigid plane through a "Method of Images" approach, in which the original quadrupole is replaced by a quadrupole system comprising the original quadrupole plus an 'image quadrupole' representing the image of the original in the plane rigid boundary. Extending this work, predictions were made of some of the basic aeroacoustic characteristics associated with a plane two-dimensional wall-jet.

Subsequent work by Davies [18] on high-frequency acoustic quadrupoles in close proximity to a rigid sphere indicates that, provided the sources are close enough to the surface, it can be regarded as locally plane, since only a small additional component is introduced as a result of its curvature. This means that the theory developed previously for a plane twodimensional wall-jet can fairly readily be extended to the case of a three-dimensional flow over a rigid Coanda surface. It was found [12-15] that the relatively simple 'Randomly Orientated Longitudinal Quadrupole' (ROLQ) method of modeling the acoustic sources gives good agreement with the results obtained by Ribner's much more complicated method of source modeling. In this work it is assumed that the zone of peak turbulence dominates the sound generation. Results obtained from this work are presented in Section 2.

\subsubsection{Shock-Associated Noise (SAN)}

The flow under consideration in the current work is that associated with a turbulent Coanda flare of the type shown in Figure 1. Green [19] has shown that one-dimensional flow theory can be used to describe the flow through a convergent-divergent nozzle of the kind present in the Coanda flare. Thus, the jet emerging from the exit slot is supersonic for almost all operating pressures. In contrast to subsonic jets, conditions at a downstream point in a supersonic jet cannot affect those upstream. In this way, discontinuities in flow properties can arise. Depending upon the relative pressure difference between the nozzle exit pressure $\left(\mathrm{p}_{\mathrm{e}}\right)$ and the ambient pressure $\left(\mathrm{p}_{\mathrm{a}}\right)$ a stationary shock-cell structure is formed in the mixing layer region close to the jet exit slot. This effect can be seen in the Schlieren flow visualization photograph of Figure 3. The exact location of these shock-waves depends upon a variety of factors including the relative magnitudes of the pressure in the reservoir supplying the nozzle $\left(\mathrm{p}_{0}\right)$ and the pressure of the medium into which the jet flows, known as the ambient or back pressure $\left(\mathrm{p}_{\mathrm{a}}\right)$. 


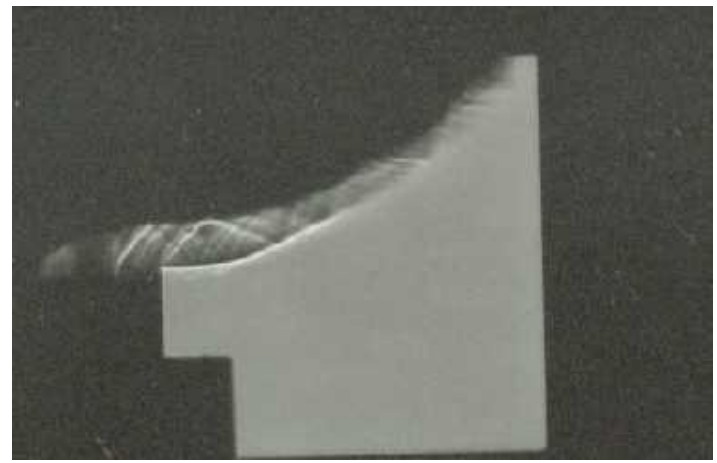

Figure 3: Typical flow structure in Coanda flare mixing layer.

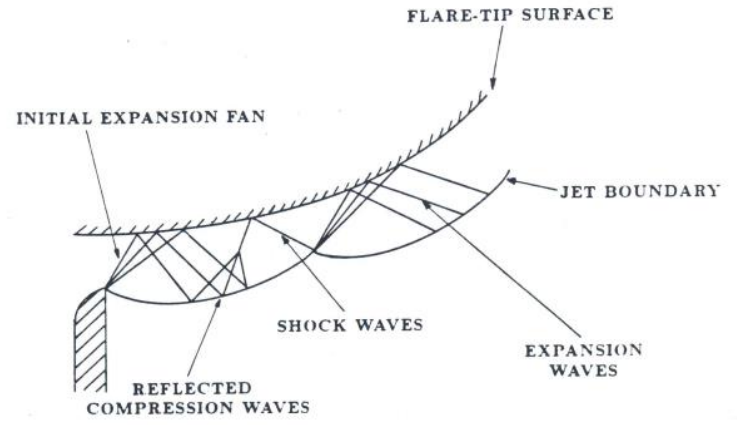

Figure 4: Shock-cell structure near the exit slot of the flare (reproduced from [12])

In the case of the flare jet, a pattern of expansion, compression and shock-waves is present under a wide range of operating conditions. The quasi-periodic constituents of this pattern, known as shock cells, are shown schematically for a Coanda flare jet in Figure 4. Typically a series of 8-10 shock cells will form in the jet exhaust. Turbulent eddies convected downstream within the mixing layer region of the jet cause these shocks to be deformed. This distortion of the shock front propagates away as broadband, but strongly peaked high-frequency sound waves known as Shock-Associated Noise (SAN). Shock-associated noise has several interesting aspects. Firstly there is a strong directivity associated with the SAN emitted by moving sound waves. It has been observed [25] that a turbulent eddy can successively interact with several shock waves, generating multiple sound sources (one resulting from each interaction). Additionally, one or more feedback cycles is often present, leading to the generation of discrete, harmonically related tones.

The Coanda flare under investigation is two-dimensional, convergent-divergent and supersonic under most operating conditions. It is well known that the flow just downstream of the jet exit slot is responsible for most of the flare noise generation and that most of the SAN is produced in his region [19]. Thus in order to fully comprehend this high-frequency acoustic emission, it is very important to understand the behaviour of the flow in the initial region near the nozzle exit. Green has previously shown that one-dimensional flow theory and the method of characteristics can be used to describe flow through this nozzle, at least until the first shock is formed [14][19-21]. The method of characteristics has previously been applied[22][23] to the inviscid core of a supersonic jet (following the method of Dash et al [24] whereby the outer shear layer and surface boundary layer are ignored) where the aim was to better predict jet breakaway. Indeed, comparison of predictions with experimental results for the location of the first shock cell (at low blowing pressures and before any shock waves occur) is good. Specifically, according to Gilchrist and Gregory-Smith, "The method of characteristics is shown to be adequate for calculating the [shock cell structure in the] inviscid core of the underexpanded [flat] jet"[23]. In the current work, it is the location of the first shock cell that is of interest, since "for high speed jets, the shock-cell structure close to the slot is very important"[23].

The shock cell structure associated with a turbulent Coanda wall jet is estimated using a standard Method of Characteristic approach. The three governing partial differential equations (speed of sound, irrotationality, gas dynamic) are rewritten in terms of two non-linear ordinary differential equations; the characteristic equation and the compatibility equation. The Euler predictor-corrector numerical integration algorithm is then used to rewrite these equations as finite difference equations and solve them at points in the flare jet flow field immediately downstream of the nozzle exit. In this way, the shock cell structure associated with a particular turbulent Coanda jet is estimated, and these predictions are compared with experimental results obtained using flow visualisation techniques. This phenomenon has previously been found to be particularly important in determining both the OASPL and peak frequency of the broadband 
high-frequency Shock-Associated Noise (BBSAN) emitted by a given jet configuration. The current work has illuminated the relationship between cell location and flow characteristics, and thus the effect of jet operating conditions on BBSAN can now be determined [25]. The relationship between cell location and jet breakaway is also under investigation. This work is in the process of being extended so that the Rankine-Hugoniot conditions can be used to predict the shock cell structure (and thus the BBSAN) along the entire jet [26].

\section{RECENT EXPERIMENTAL RESULTS}

\subsection{Introduction}

Experiments were conducted in a $5 \mathrm{~m} \times 2.5 \mathrm{~m} \times 2.5 \mathrm{~m}$ anechoic chamber. The model consisted a "tulip-shaped" body of revolution with an annular nozzle leading to an exit slot at the base of the tulip. Some models were fitted with a step at the base of the tulip (so-called 'stepped flares'). Fitting such a step has the effect of postponing jet detachment, or breakaway, to higher operating pressures, but it also leads to other complications. The addition of a step undoubtedly makes the flow field and acoustic characteristics significantly more complex. The Coanda surface had a $53.3 \mathrm{~mm}$ maximum diameter, where $R_{c}$ (radius of the flare center stem) $=18.056 \mathrm{~mm}$ and $R_{f}$ (radius of the spherical section of the flare) $=9 \mathrm{~mm}$. The jet exit velocities $\left(\mathrm{U}_{\mathrm{jx}}\right)$ were between $200 \mathrm{~m} / \mathrm{s}$ and $500 \mathrm{~m} / \mathrm{s}$, and the exit slot $(\mathrm{h})$ varied from $1.14 \mathrm{~mm}$ to $4.19 \mathrm{~mm}$. All experiments were carried out at ambient (room) temperature and pressure. Simultaneous sound measurements (using four B \& $\mathrm{K} 1 / 4$ inch condenser microphones at angles of $11.3^{\circ}(\mathrm{mic} 1), 5^{\circ} 7^{\circ}$ (mic 2), $0^{\circ}(\operatorname{mic} 3)$ and $-6^{\circ}$ (mic 4) to the horizontal) and Schlieren flow visualization experiments were conducted as shown in Figure 5. A time-synchronized digital video camera was used to record the experiments and selected frames were used to investigate flow behaviour. Shock-cells formation was observed under almost all operating conditions.

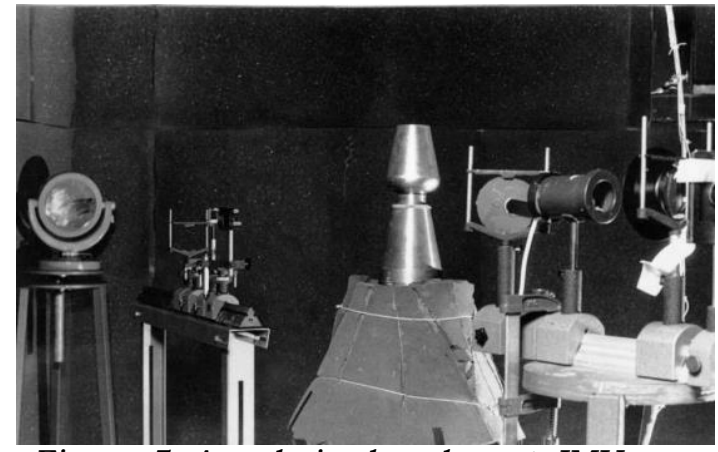

Figure 5: Anechoic chamber at JMU

A typical Schlieren image was shown in Figure 3 and the associated noise spectrum is shown in Figure 6.

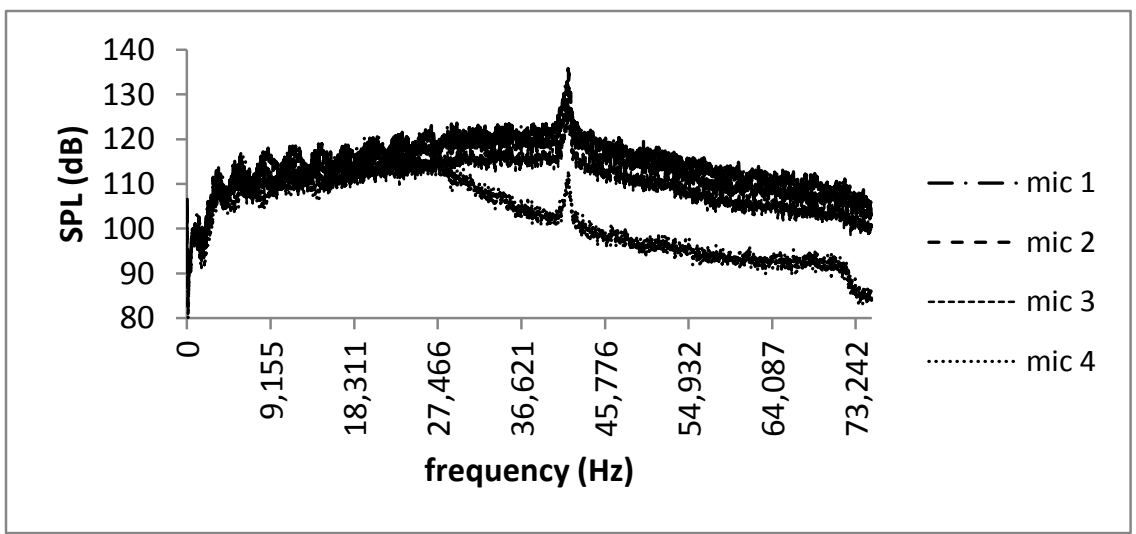

Figure 6: Flare spectrum (54mm diameter, $2.39 \mathrm{~mm}$ slot width, operating pressure 35 psig) 


\subsection{Results}

Breakaway is the phenomenon that is often observed in Coanda flows when a lip shock is formed at the lower edge of the exit slot. This lip shock generates a separation bubble on the Coanda surface which grows in size with operating pressure and ultimately causes the flow to separate from the Coanda surface to which it was formerly attached [14]. The breakaway process is shown in more detail by the set of Schlieren photos in Figure 7(a)-(d). For the Coanda surface studied, this occurs at certain slot width-operating pressure (or exit velocity) combinations, as shown in Figure 8. Figure 9 shows how the SPL is affected by breakaway. As soon as breakaway occurs, a steep drop in SPL is observed as the flow is redirected away from the horizontal.

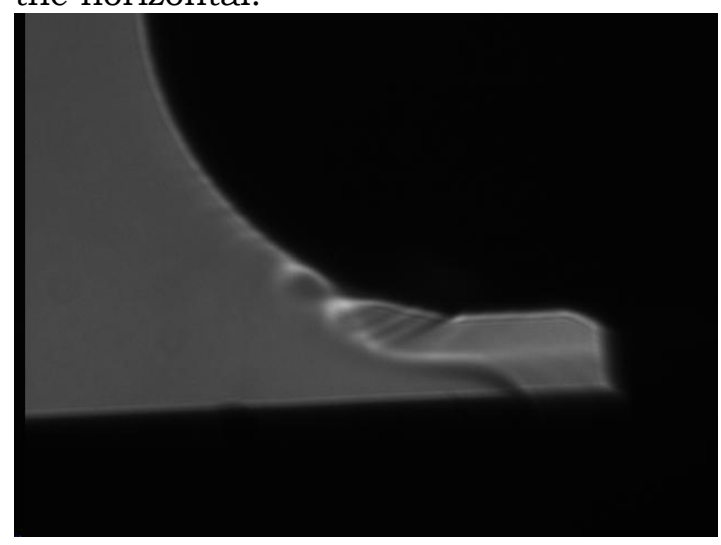

(a) $45 p$ sig

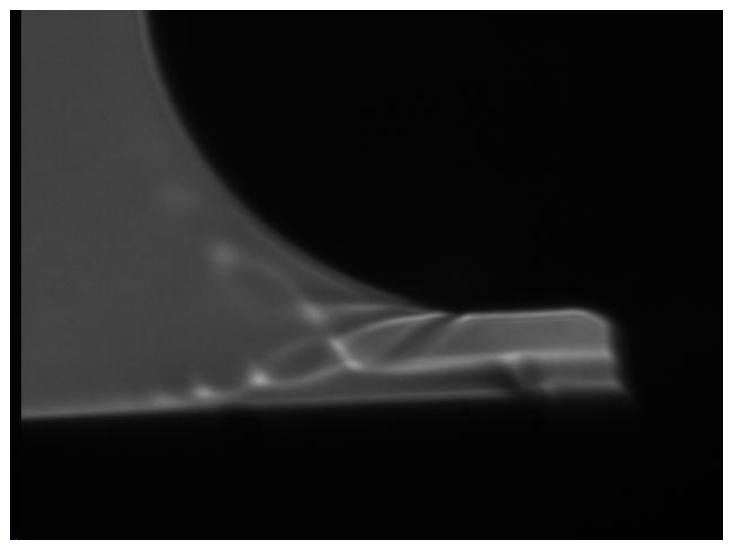

(c) During breakaway

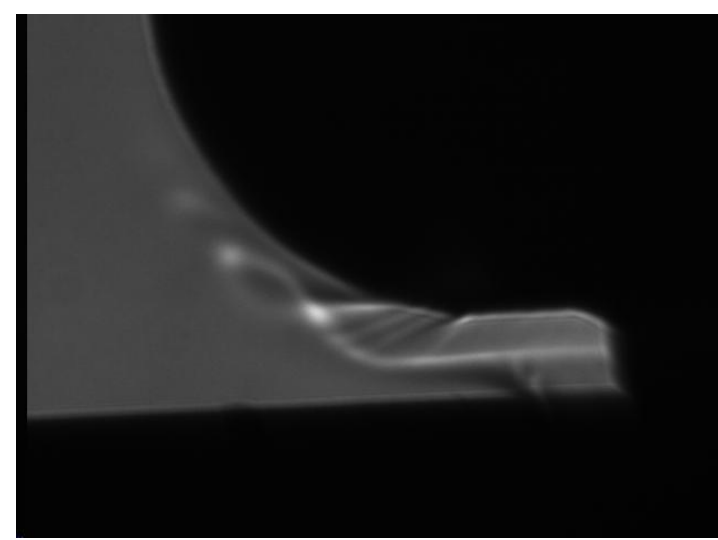

(b) Before breakaway

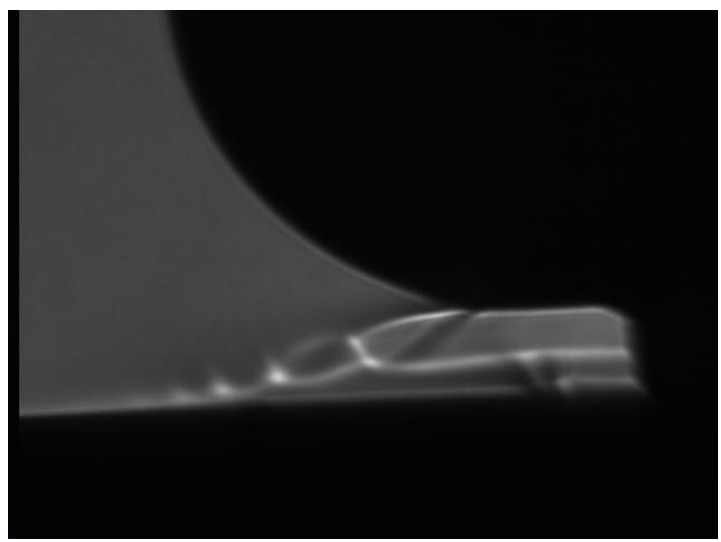

(d) After breakaway

Figure 7: Schlieren photographs showing breakaway, $2.82 \mathrm{~mm}$ slot width

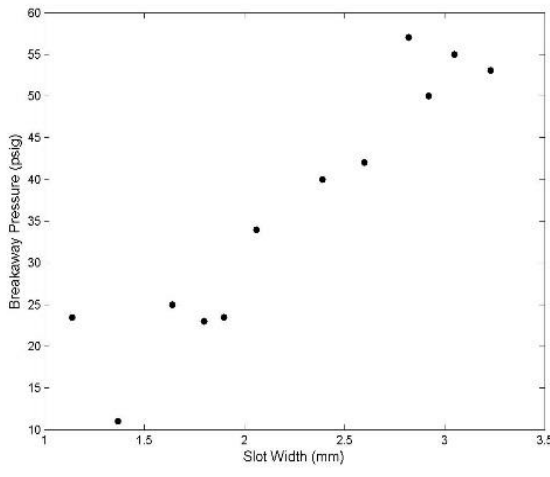

Figure 8: Variation in breakaway pressure with slot width

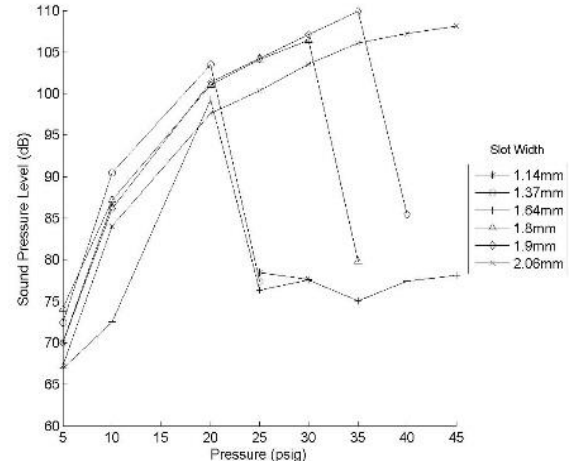

Figure 9: Effect of breakaway on SPL 
The relationship between breakaway and the observed shock cell structure and operating characteristics is currently being investigated.

In order to prevent breakaway, or at least delay it to well above maximum operating pressures, a step was introduced at the base of the tulip-shaped body of revolution. This step precluded formation of the lip-shock and related separation bubble, thereby preventing the associated breakaway. However the presence of this step does lead to the development of a stagnant cavity between the base of the flare and the step, as shown in Figure 10.

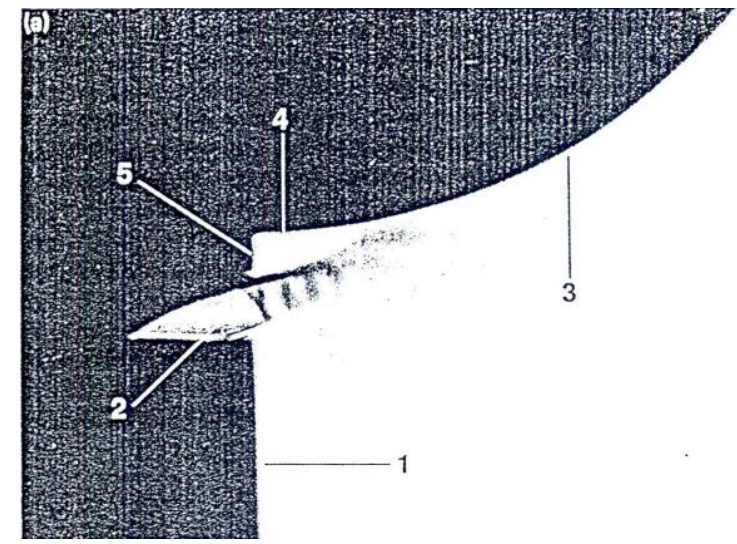

Figure 10: Inclusion of step (5) at the base of the tulip-shaped body of revolution (1 and 3). A stagnant cavity can be seen at 4, and the associated shock cell structure at 2.

The sound pressure level (OASPL) directivity was investigated experimentally for both a stepped and unstepped I4 Coanda flow and the results are shown in Figure 11. Clearly the step causes a steep rise in the maximum OASPL and also strongly affects the directivity pattern. In particular, for the current configuration of operating conditions it shifts the maximum OASPL to below the horizontal, although this is not always the case with other configurations. The irregular pattern in Figure 11(b) when the pressure ratio is 2.4 is due to the presence of discrete tones in the flow for that pressure ratio. It is postulated $[14,15\}$ that these are generated by a self-excited acoustic feedback loop caused by the interaction between the stagnant cavity and the large-scale coherent structures in the flow. Discrete tones were generated throughout the range of operating conditions and their presence had the effect of disrupting observed trends.

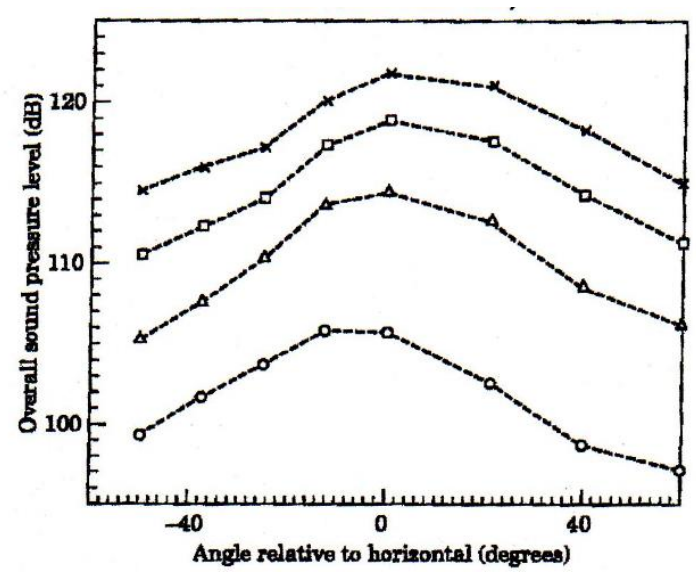

(a)

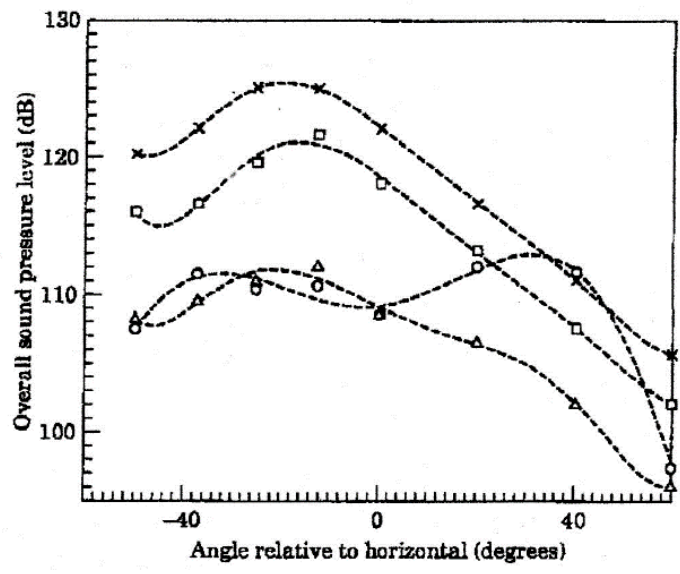

(b)

FIGURE 11: Directivity of the OASPL (a): unstepped I4 (Reproduced Fig. 10, ref. [14]); (b): stepped I4, with $2 \mathrm{~mm}$ step (Reproduced Fig. 4, ref. [15]). The directivity patterns are plotted for four different pressure ratios (ratio of stagnation to ambient pressure), namely $\bigcirc 2.4 ; \Delta 3.8 ; \square$ 5.2; and $\mathrm{X}$ 6.6. 
For round jets, the OASPL follows Lighthill's well-known $\mathrm{U}_{\mathrm{jx}}{ }^{8}$ law. From Figure 12(a) it is clear that the unstepped data also follows this law. However, from Figure 12(b) for the stepped flare, no clear trend can be discerned.

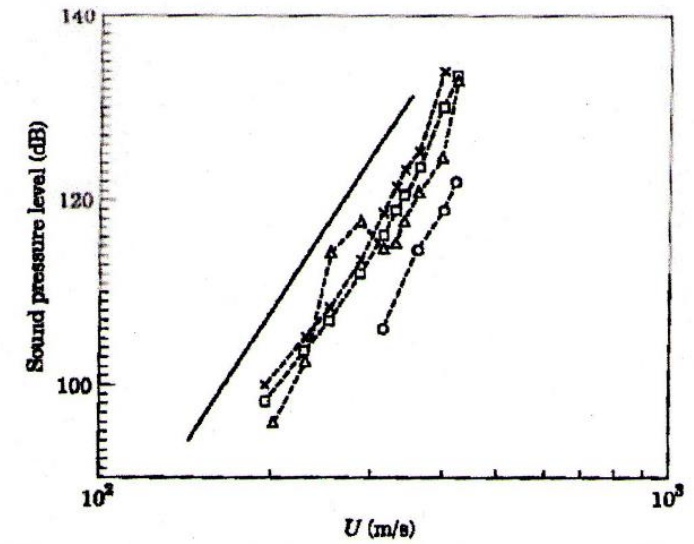

(a)

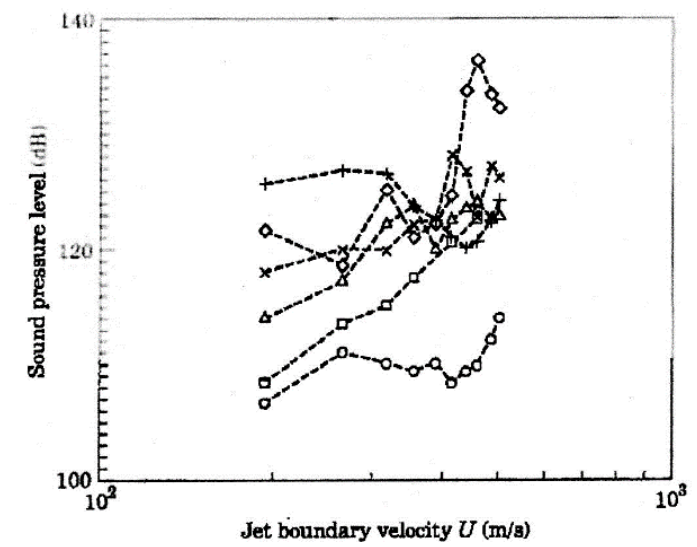

(b)

FIGURE 12: OASPL versus Ujx (a): unstepped I4 (Reproduced Fig. 10, ref. [14]); (b): stepped I4, with $2 \mathrm{~mm}$ step. (Reproduced Fig. 4, ref. [15]). The lines represent different slot widths as follows: $\bigcirc h=0.625 \mathrm{~mm} ; \Delta h=1.25 \mathrm{~mm} ; \mathrm{X} h=1.875 \mathrm{~mm} ; \diamond h=2.5 \mathrm{~mm} ;+h=3.125 \mathrm{~mm}$. The solid line on Fig. 12(a) represents Lighthill's $U_{j x} 8$ law.

Figure 13 shows how the primary tone frequency of the discrete tones described above varies with $\mathrm{U}_{\mathrm{jx}}$ (via pressure ratio) for different slot widths, h. The clear division of the data into two groups, A and B, reflects that in Group A all the data are at relatively low stagnation pressures and that frequency is almost invariant with slot width, whereas Group B data are at higher stagnation pressures and frequency tends to decrease with pressure. The unstepped and stepped results are intermingled in this figure. Both sets of tones are thought to be established around self-excited feedback mechanisms. However, those in Group A are primarily based on the stagnant cavity, whereas those in Group B are generated by the separation bubble. In the case of the Group B tones, there are four distinct stages, each characterized by a sharp jump in frequency, followed by a subsequent drop in frequency with a further rise in stagnation pressure.

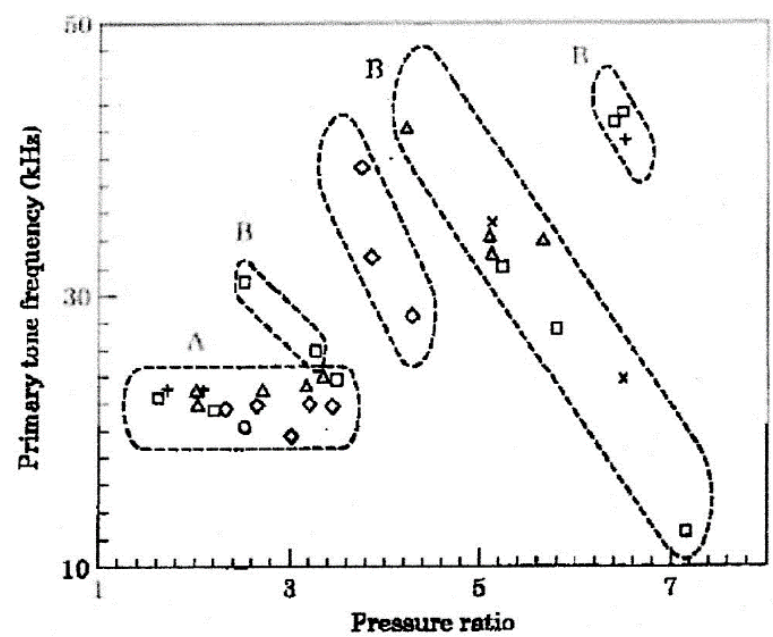

FIGURE 13: Primary tone frequency versus pressure ratio for various slot widths. (Reproduced Fig. 8, ref. [15]). Unstepped slot widths: $+h=1.25 \mathrm{~mm} ; \mathrm{X} h=1.875 \mathrm{~mm}$; slot widths for $2 \mathrm{~mm}$ step; $\bigcirc \mathrm{h}=3.125 \mathrm{~mm} \diamond \mathrm{h}=2.5 \mathrm{~mm} ; \Delta h=1.875 \mathrm{~mm} ; \square \mathrm{h}=1.25 \mathrm{~mm}$.

Based on the postulated mechanism for discrete tone generation, a technique for eliminating such tones was proposed in which a saw-toothed sleeve was fitted to the annular 
exit nozzle of a stepped flare (Figure 14(a)). The saw-teeth generated longitudinal trailing vortices (Figure 14(b)) which disrupted the large-scale coherent eddy structure present in the jet, thereby destroying the self-excited cavity-eddy feedback loop and completely eliminating the associated discrete tones.
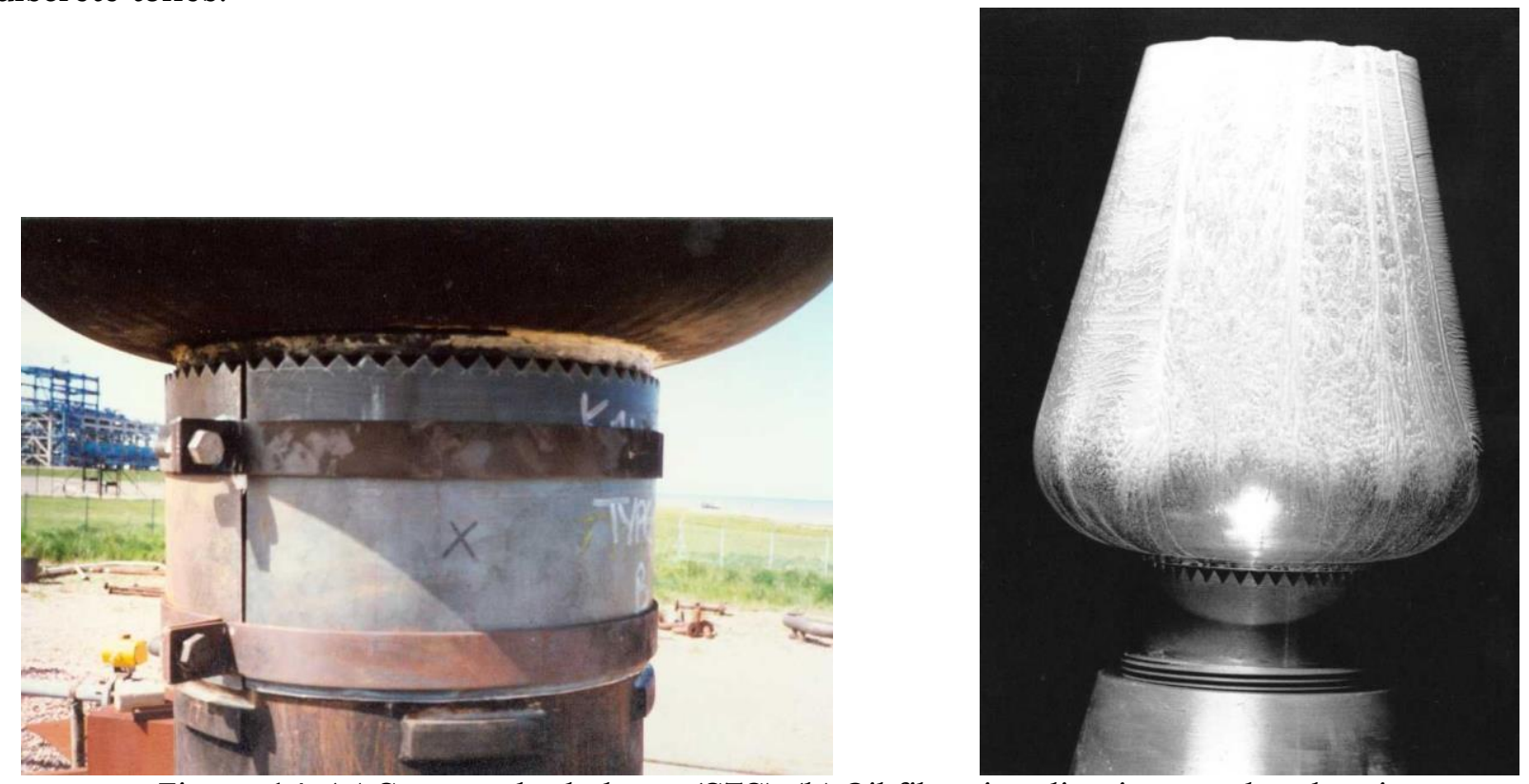

Figure 14: (a) Saw-toothed sleeve (STS) (b) Oil film visualization results showing longitudinal vortices generated by use of STS

Figure 15 shows the predicted shock-cell pattern superimposed on the previously described experimental results, for $\mathrm{U}_{\mathrm{j}_{\mathrm{x}}}=467 \mathrm{~m} / \mathrm{s}$, and $\mathrm{h}=3.05 \mathrm{~mm}$. Other predictions exhibit similar features. The arrows represent the $(\mathrm{u}, \mathrm{v})$ vectors at each point predicted by the intersections of characteristics, starting with the flow in the exit plane as well as the initial expansion fan. Shock waves are formed at the coalescence of these vectors and both the dark regions on the flow visualization figures, and the higher concentration of vectors (shown in white) correspond to regions of higher pressure.

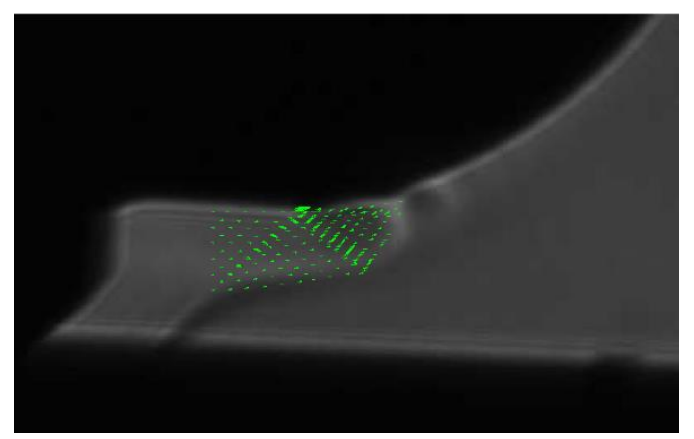

(a)

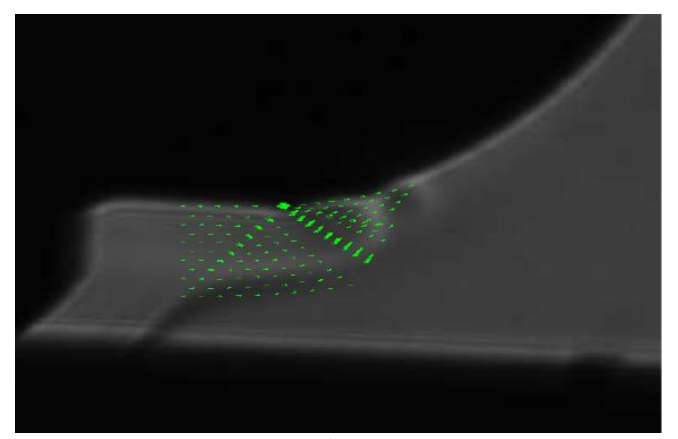

(b)

Figure 15: Comparison of experimental \& theoretical results. (a) $h=2.82 \mathrm{~mm}$, 30psig, (b) $h=3.23 \mathrm{~mm}, 35 p s i g$.

Comparison indicates that the preliminary model of SAN is relatively accurate at predicting the location of the first shock cell formation. Cells further from the exit slot are less well predicted, and future work will focus on modifying this preliminary model to include radial expansion and streamline curvature, which is anticipated to improve these predictions. 


\subsection{Comparison of Coanda flows with plane/axisymmetric flows}

In the case of a Coanda jet flow field, there are many complicating factors compared with plane or axisymmetric flows. These include radial expansion and streamline curvature, both of which will lead to considerably higher levels of turbulence than in the mixing layer of a plane jet. Although the growth rate of the mixing layer has been found to be similar in both cases, the potential core is generally shorter in the case of Coanda flows, because radial expansion causes the inviscid boundary layer to move towards the surface in order to conserve mass flow. It is found that the effects of source convection are much more important for a wall jet than a free jet. When discrete tones are absent, the SPL is proportional to both $\mathrm{U}^{8}$ and $\mathrm{h}^{2}$ (as expected following Lighthill). However, an unexpected result is that the broadband peak frequency is a function of the maximum Coanda diameter (rather than the traditional Lighthill characteristic length scale of slot width, h).

\section{APPLICATION TO LAUNCH ACOUSTICS}

The work described in the following sections (Sections 3.1 - 3.4] was conducted by Panda, Mosher and Porter on behalf of NASA and Orbital Sciences Corporation. It has been reproduced here with the kind permission of NASA and Orbital. For full details of the experimental work, and other related work, please see [30]. This reference also acknowledges the many other people involved in producing these results.

\subsection{Introduction}

During lift-off, a launch vehicle and its environs are subjected to extremely high, fluctuating acoustic loads. These loads are a principal source of structural vibration and can critically affect correct operation of the launch vehicle, the vehicle components and supporting structures. Even small reductions in this noise level can lead to significant savings in terms of unexpected repairs, operating costs and system failures. Thus a research project was recently undertaken to identify the primary noise sources generated during the launch of the Orbital Sciences Antares rocket. The Antares rocket is one of two vehicles currently contracted by NASA to resupply the International Space Station (ISS). (The other is SpaceX's Dragon). Such an investigation has not previously been undertaken during an actual rocket launch, except for an experiment in which twelve microphones were placed on the flight vehicle rather than the launch pad [30].

In order to identify the level and location of the various noise sources, a phased-array of 70 GRAS $1 / 4$ inch flush-mounted condenser microphones (in a 10 foot by 10 foot ( $3 \mathrm{~m}$ by $3 \mathrm{~m}$ ) frame) was used. The array was mounted on a scissor lift raised $40(12 \mathrm{~m})$ feet above the ground, at a distance of 400 feet $(122 \mathrm{~m})$ from the pad. The microphone array is shown in detail in Figure 16.

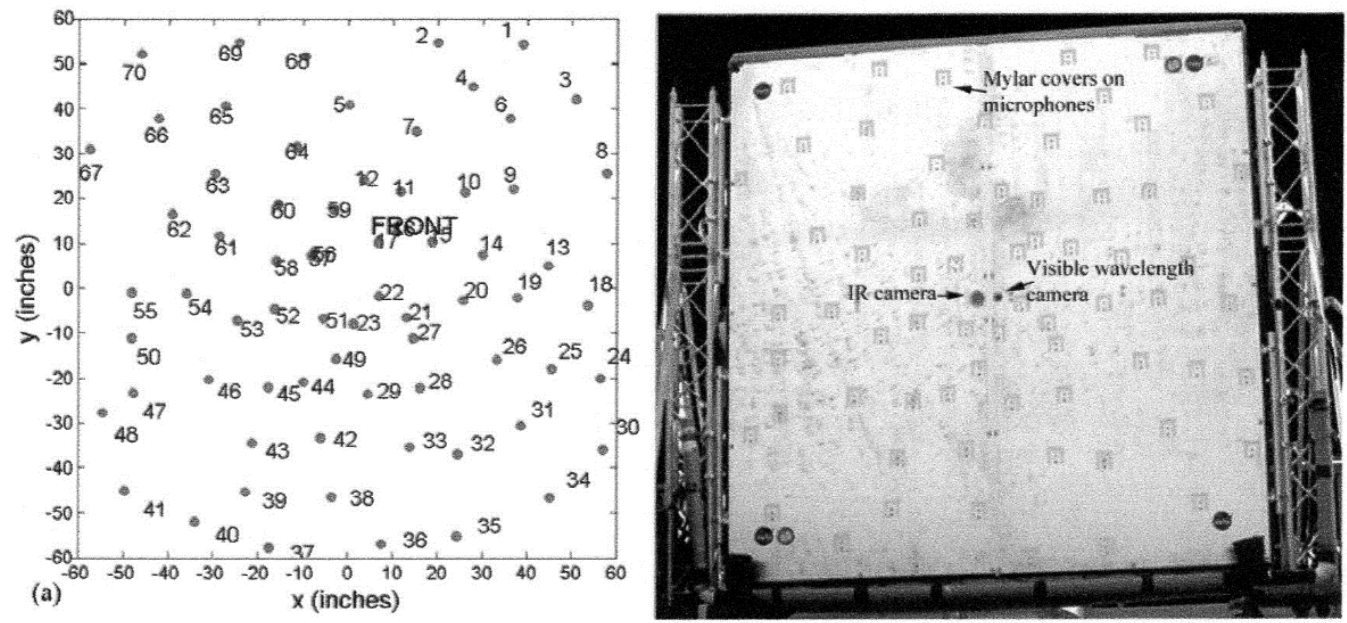

Figure 16: (a) Microphone layout and (b) a photograph of the front surface of the 10 foot by 10 foot array. (Reproduced from Fig. 3, ref. [30]). 
The goal of this particular microphone layout was to maximize the array resolution at the lower frequency range $(400 \mathrm{~Hz}-2 \mathrm{KHz})$ whilst also reducing the side lobes. The microphone array also houses a two-axis accelerometer and two cameras at its center (a 1.3MPixel visible wavelength camera and a 640 by 480 Pixel infrared wavelength thermal camera). More details of the array are given in [30]. The acoustic suppression system already in place on the specially-built Antares launch pad (located in Wallops Island, Va) is composed of three separate sets of water injection ports as shown in Figure 17.

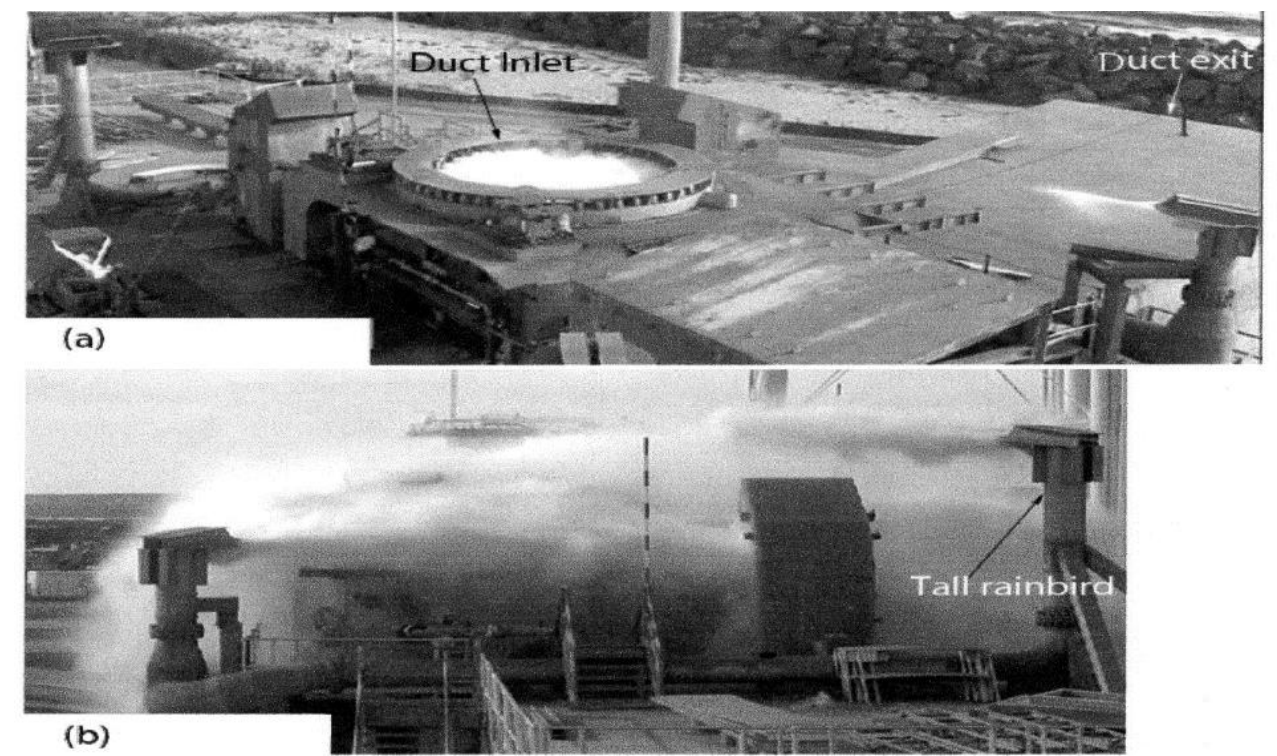

FIGURE 17: Water injection systems used for acoustic attenuation in launch pad OA. (a) Duct-inlet water; (b) on-deck 'rainbird' water). (Reproduced from Fig. 2, ref. [30])

These comprise the 'duct inlet water', the 'J-deflector water' and the 'rainbirds' (tall and short). The first two sources start to flow before engine ignition, whilst the rainbirds, which spray water onto the launch pad surface to prevent plume impingement damage, start at two different times. The shorter ones start just before the taller ones, to avoid deluging the launch vehicle.

\subsection{Phased Array Data Processing}

A full description of the phased array data processing is given in [30].

\subsection{Experimental Considerations}

The microphone phased-array set-up described above was deployed at the inaugural launch of the Antares rocket on April 21st, 2013. As a result of issues with the thermal camera during previous experiments, an improved vibration mount was used, which meant that the thermal camera provided images for about 10 seconds after launch. Unfortunately, six of the seventy microphone channels were accidentally turned off, so that the microphone array consisted of 64 microphones. However, this was perfectly adequate for providing useful data. The 'look angle' of the cameras was determined experimentally and used to correct the results from both cameras. The rocket was sufficiently slow-moving that no Doppler-correction was necessary in the beamforming calculations. However, the delay in propagation of the sound waves from the launch pad to the face of the array needed to be taken into account.

Previous launch noise prediction models have been based on the notion of the plume as the primary noise source throughout launch. However in this work, which represents the first time a ground-based beamforming experiment has been conducted during an actual launch, the noise sources during launch are found to be time-dependent. Figure 18 clearly shows this time evolution. 

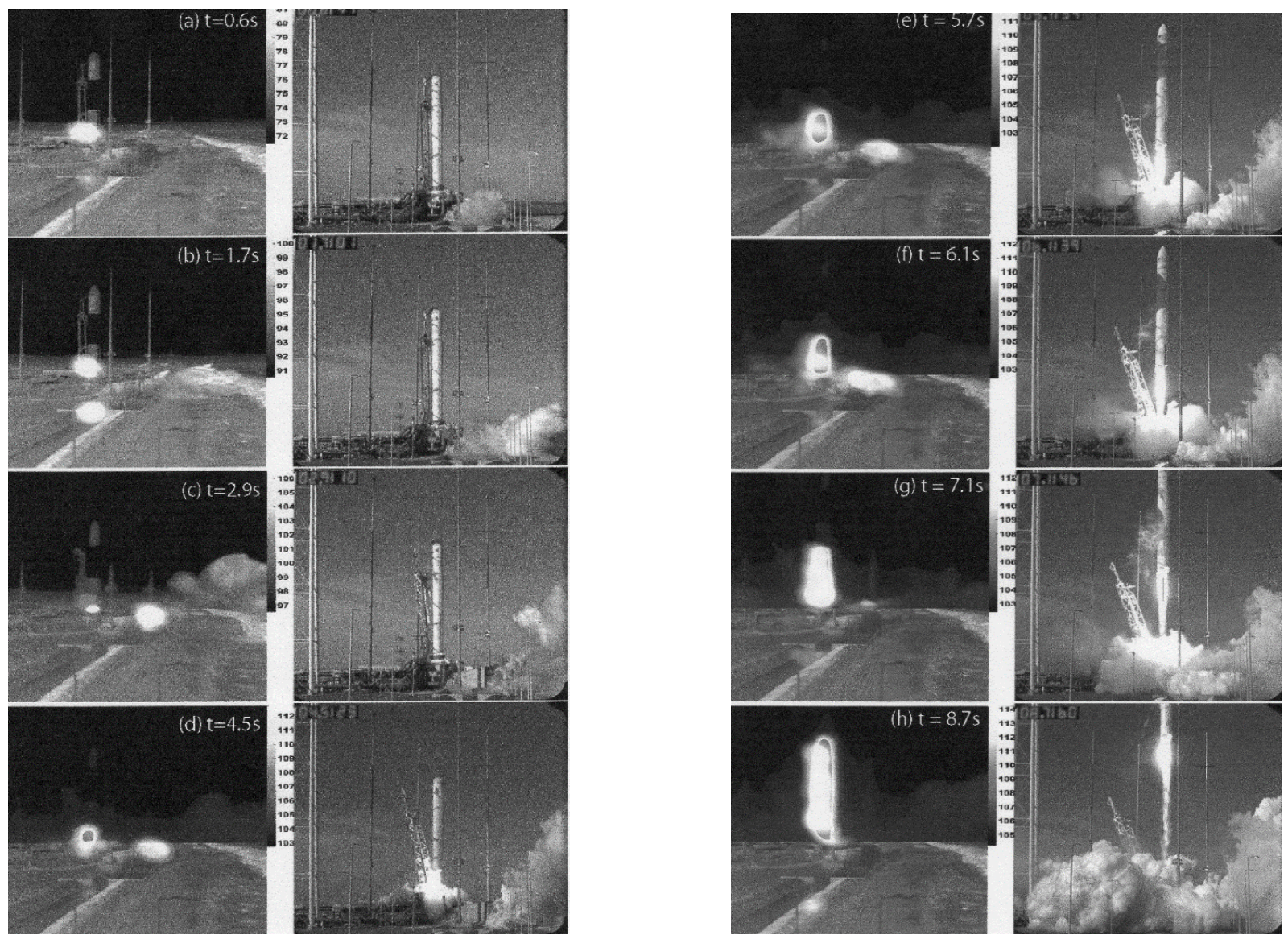

Figure 18: Left column noise maps superimposed on frames from IR camera, conventional beamform at $2 \mathrm{kHz}$; (right) corresponding frames from a high-speed camera. (Reproduced from Fig. 11, ref. [30]).

The left-column pictures are composites of three separate images; this is necessary to provide enough definition of the pad, which was obscured by steam, water and debris generated during launch. The three images are (a) a beamformed noise map whose levels are shown in color; (b) the bottom section of a temperature map from just prior to ignition; and (c) the top section of the temperature map at the time shown on the Figure. The right-hand column images (selected frames from a high-speed camera) are courtesy of NASA KSC imaging group. In these Figures, the Transporter Erector Launcher (TEL) can be seen releasing the launch vehicle and pitching away from the vehicle early in the launch sequence, as the launch vehicle simultaneously fires at an slight angle to the vertical away from it; the so-called 'TEL avoidance maneuver'. The significantly affects the noise distribution, because the tilting of the vehicle, combined with its motion, causes the hot exhaust plume (responsible for most of the noise) to spill out from the duct inlet (designed to suppress the associated noise) and spread across the launch pad. The observed start of the rainbirds (later than initially expected) compounded this problem.

A practical application of the work described previously (concerning turbulent Coanda jet flows) is to launch acoustics. The recent experimental data (summarized in Section 3.4) has indicated that a thorough understanding of the changes in acoustic source location with launchphase is of fundamental importance. During this time-dependent behavior, the flame duct -- or $\mathrm{J}$-deflector -- is a fundamental player in noise generation. 


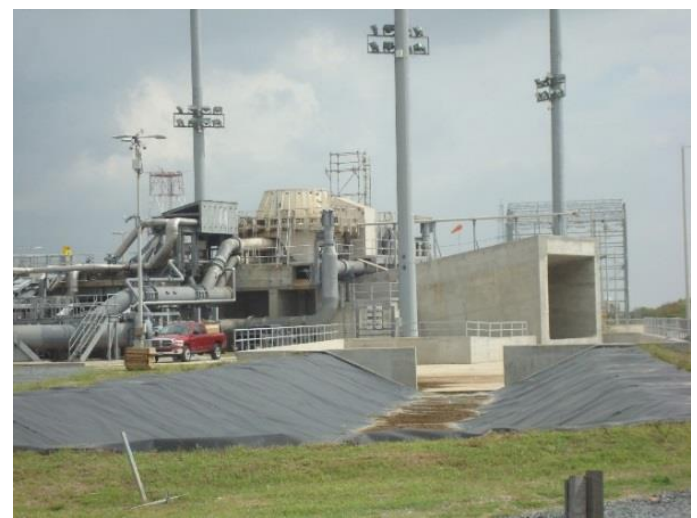

(a) Photograph of J-deflector

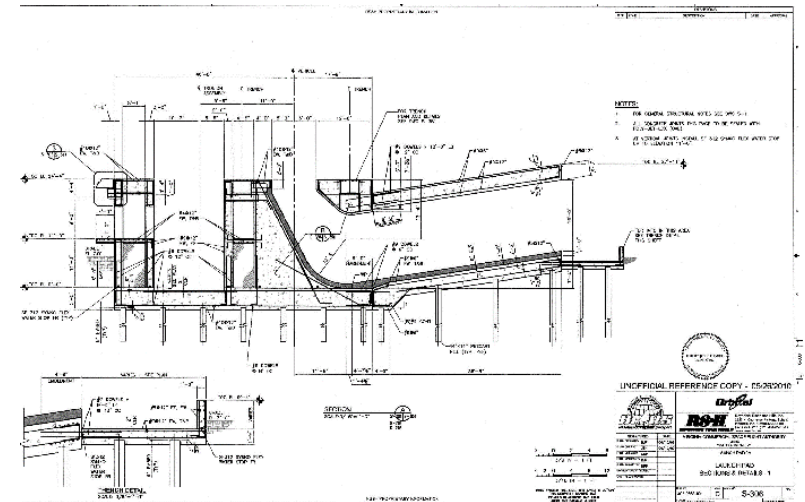

(b) Technical drawing of J-deflector

Figure 19: Antares rocket flame trench (J-deflector), Wallops Island, Va.

This J-deflector, shown in Figures 19 (a) and (b), is the primary noise source as the engines come to full power, at a time when acoustic-induced vibration is potentially very damaging to the launch vehicle and its contents. More specifically, during this period the primary noise source changes from being the duct inlet to the duct exit. Applying some of the results obtained previously (outlined in Sections $2.1-2.5$ ) will hopefully lead to a greater understanding of the turbulent wall jet flow within this deflector (which is a Coanda flow, as shown in Figure 19(b)) and might significantly contribute to noise reduction during this phase of the launch process. The benefits of reduction of acoustic load at launch have already been elucidated in Section 3.1. This work is currently underway.

\subsection{Results}

Data collected from the experiments described in the previous sections provided information not previously available. In particular, information was obtained pertaining to acoustic sources during a full-scale launch which indicated that the source distribution was very different from that used in traditional models of lift-off acoustics. These sources were found to be time-dependent, and to depend strongly on the phase of the launch. Initially, during engine ignition (just prior to lift off) the primary noise source was the launch mount (duct inlet). However, once the engines came to full power, the hot exhaust plume exited the duct and the duct exit (J-deflector exit) became the primary source, although this effect was mitigated to some degree by the duct water. As the launch vehicle started to tilt and rise, the motion away from the duct caused the plume to spill across the launch pad and a large area on the top surface of the pad became a loud, distributed acoustic source, although initiation of the rainbirds reduced its extent. As the rocket gained vertical elevation, the plume itself became the primary noise source. At this point, the water suppression system was at full power and the rainbirds were still cooling the top of the pad, but not the plume itself, of course. Ground reflection was identified as the primary noise source only at ignition and when the plume emerged from the duct, but not in the interim.

\section{SUMMARY AND CONCLUSIONS}

A theoretical method of predicting the turbulent mixing noise and shock-associated noise (via the shock-cell structure) associated with a three-dimensional turbulent Coanda wall jet has been presented, and compared with experimental data. Initial agreement is reasonable, for the operating conditions considered. Some general trends in behaviour are observed. The location of the first shock cell appears to be unaffected to any large extent by jet exit velocity, and to decrease with slot width. The presence of discrete tones in the data disrupt these trends. Breakaway is achieved under certain operating conditions and the relationship of this phenomenon to the observed shock waves is currently being investigated in more detail. A comparison of these results with those of plane or axisymmetric flows highlights the difference effects that occur when dealing with Coanda wall jets. 
A turbulent Coanda wall jet of current interest is the one which occurs in the flame trench used during launch of the Antares rocket. Recent experimental data has indicated that a thorough understanding of the changes in acoustic source location with launch-phase is of fundamental importance. During this time-dependent behavior, the flame duct, or J-deflector, is a fundamental player in noise generation. Specifically, it is the primary noise source when the engines reach full power, at a time when acoustic-induced vibration is potentially very damaging to the launch vehicle and its contents. A thorough understanding of this Coanda flow should contribute significantly to noise reduction during this phase of the launch process. Methods developed during the previous work on turbulent Coanda wall jets are currently being applied to this flow.

\section{REFERENCES}

1. Coanda,H., Procede de propulsion dans un fluide, Brevet Invent. Gr. Cl. 2, no. 762688 Republique Francaise, (1932).

2. Lubert, C.P., On Some Recent Applications of the Coanda Effect, Int. J. Acoustics and Vibration, 16(3), 144-153, (2011).

3. Lubert, C.P, Application of Turbulent Mixing Noise Theory to Flows over Coanda Surfaces, Int. J. Acoustics and Vibration, 13(1), pp17-30, (2008).

4. Desty, D.H. No smoke without fire, Proceedings of the Institution of Mechanical Engineers, 197A, 159-170, (1983).

5. Desty, D.H, Boden, J.C. and Witheridge, R.E. The origination, development and application of novel premixed flare burners employing the Coanda effect, 85th Nat. AIChE meeting, Philadelphia, (1978).

6. Lighthill, M.J., On sound generated aerodynamically. I. General Theory, Proc. Roy. Soc., 211, pp564-587, (1952).

7. Lilley, G.M., On the Noise from Air Jets, ARC Report 20,376, Aeronautics Research Council, UK, (1958).

8. Ribner, H.S., On the Role of Shear Noise, J. Sound. Vib., 51(1), pp121 - 132, (1977).

9. Parsons, C., An experimental and theoretical study of the aeroacoustics of external-Coanda gas flares, Ph.D. Thesis, University of Exeter, (1988).

10. Smith, C., and Carpenter, P.W. The effect of solid surfaces on turbulent jet noise, J. Sound and Vibration, 185(3), 397-413, (1995).

11. Carpenter, P.W., and Smith, C. The aeroacoustics and aerodynamics of high-speed Coanda devices, Part 2: Effects of modifications for flow control and noise reduction, J. Sound and Vibration, 208(5), 803-822., (1997).

12. Parsons, C., (maiden name of C. Smith/Lubert) An experimental and theoretical study of the aeroacoustics of external-Coanda gas flares, Ph.D. Thesis, University of Exeter, 1988.

13. Parsons, C., (maiden name of C. Smith/Lubert) and P.W. Carpenter, 1986, AIAA Paper 861869, Proc. American Institute of Aeronautics and Astronautics, Seattle, USA. The Noise Emitted by Turbulent Jets in Close Proximity to Solid Surfaces. 
14. Carpenter, P.W., and Green, P.N., The Aeroacoustics and Aerodynamics of High-Speed Coanda Devices, Part 1: Conventional Arrangement of Exit Nozzle and Slot, J. Sound and Vibration, 208(5), pp 777-801, (1997).

15. Carpenter, P.W., and Smith, C., The Aeroacoustics and Aerodynamics of High-Speed Coanda Devices, Part 2: Effects of Modifications for Flow Control and Noise Reduction, J. Sound and Vibration, 208(5), pp 803-822, (1997).

16. Curle, N., The Influence of Solid Boundaries upon Aerodynamic Sound, Proc. Royal Soc., A231, pp. 505-514, (1955).

17. Powell, A., Aerodynamic noise and the plane boundary, J. Acoust. Soc. America, 32, 982-990, (1960).

18. Davies, H.G., The radiated fields of multiple point sources near a solid spherical surface, $J$. Fluid Mech., 43, pp. 597-606, (1970).

19. Green, P.N., The Fluid Dynamics and Aeroacoustics of External Coanda Flares, PhD Thesis, Exeter, (1987).

20. Carpenter, P.W. and Green, P.N. The Generation of Noise in External Coanda-Type WasteGas Flares, Proc. I.O.A., Vol. 6, Part 1, (1984).

21. Bull, D., The shock associated noise of the Indair flare, Undergraduate project report, University of Exeter, UK, (1980).

22. Gilchrist, A.R., The development and breakaway of a compressible air jet with streamline curvature and its application to the Coanda flare, PhD thesis, Durham University, (1985).

23. Gilchrist, A.R., and Gregory Smith, D.G., Compressible Coanda Wall Jet; Predictions of Jet Structure and Comparison with Experiment, Int.J. Heat Fluid Flow, 9(3) 286-295, (1988).

24. Dash, S.M., Beddini, R.A., Wolf, D.E., and Sinha, N., Viscous/Inviscid Analysis of curved sub- or supersonic wall jets, AIAA Paper 83-1679, (1983).

25. Harper-Bourne, M and Fisher, M.J., The Noise from Shock Waves in Supersonic Jets, Proc. AGARD Conf. on Noise Mechanisms, CP-131, Paper 11, (1973).

26. Tam, C.K.W., Pastouchenko, N.N., and Viswanathan, K., Computation of Shock Cell Structure of Dual-Stream Jets for Noise Prediction, AIAA Journal, 46 (11), (2008).

27. Morrison, J.F., A Study of an Axisymmetric Wall Jet with Streamline Curvature and its Application to the Coanda Flare, PhD Thesis, University of Durham, UK., (1982).

28. Tam, C.W.K., Stochastic Model Theory of Broadband Shock-Associated Noise from Supersonic Jets, J. Sound Vib., 116, pp265 -302, (1987).

29. Tam C.W.K., and Tanna, H.K., Shock-Associated Noise of Supersonic Jets from ConvergentDivergent Nozzles, J. Sound Vib., 81(3), pp337-358, (1982).

30. Panda, J., Mosher, R.N., and Porter, B.J., Identification of Noise Sources during Rocket Engine Test Firings and a Rocket Launch Using a Microphone Phased-Array, NASA/TM-2013216625, (2013). 